\title{
Resultado de intervenção nutricional em crianças e adolescentes com fibrose cística
}

\author{
Results of nutritional intervention in children and adolescents with cystic fibrosis
}

\author{
Maria Cristina A. Gaspar ${ }^{1}$, Sônia M. Chiba ${ }^{2}$, Clóvis E.T. Gomes ${ }^{3}$, Yara Juliano ${ }^{4}$, \\ Neil F. Novo ${ }^{5}$, Fabio Ancona-Lopez
}

\section{Resumo}

Objetivo: poucos estudos têm verificado longitudinalmente a evolução do estado nutricional de pacientes com fibrose cística. $\mathrm{O}$ objetivo deste estudo foi acompanhar a evolução do estado nutricional, composição corporal e consumo de energia, macro e micronutrientes ingeridos por crianças e adolescentes, mediante intervenção nutricional, no Ambulatório de Fibrose Cística/Pneumologia Pediátrica, do Departamento de Pediatria da UNIFESP.

Métodos: a casuística constituiu-se de 18 pacientes, sendo 13 do sexo masculino e 5 do feminino, faixa etária de 0,3 a 18,4 anos. Realizaram-se 3 avaliações: no momento 1 (M1: pré-intervenção nutricional), no M2, após 6 meses, e no M3, após 12 meses. Foram analisados nos 3 momentos: o escore $\mathrm{Z}$ de peso/idade (P/I), peso/ estatura $(\mathrm{P} / \mathrm{E})$ e estatura/idade (E/I) e o cálculo do registro alimentar de 3 dias. No M1 e M3, verificou-se a composição corporal (antropometria). A conduta nutricional foi hipercalórica, hiperprotéica e houve adequação de macro e micronutrientes.

Resultados: observou-se aumento significante do escore $\mathrm{Z}$ de E/I (M1=-1,07; M2=-0,69; M3=-0,50) e de massa magra após a intervenção nutricional, sem melhora no escore $Z$ de P/E e massa gorda. Verificou-se aumento no consumo energético nos M2 (139\%) e M3 (132\%) em relação ao M1 (106\%). Houve considerável aumento no consumo de proteína, cálcio, ferro e vitamina $\mathrm{C}$ pelos pacientes. A presença de anemia ocorreu em 44,4\% (8/18) dos pacientes.

Conclusão: o aumento no escore Z de E/I e massa magra ocorreu devido a adequação no consumo de energia, após a intervenção nutricional. Não houve melhora significante no escore $\mathrm{Z}$ de P/E e massa gorda, em função do ganho de estatura e de massa magra.

J Pediatr (Rio J) 2002; 78 (2): 161-70: fibrose cística, intervenção nutricional, energia e nutrientes, estado nutricional, composição corporal.

\begin{abstract}
Objective: few studies have verified longitudinally the evolution of the nutritional status of patients with cystic fibrosis. The objective of this study is to follow the evolution of the nutritional status, body composition and energy consumption, macronutrients and micronutrients ingested by children and adolescents by means of nutritional interventions at the Clinic of Cystic Fibrosis/Pediatric Pneumology of the Department of Pediatrics of Universidade Federal de São Paulo.
\end{abstract}

Methods: 18 patients were involved in this study, thirteen males and five females with ages ranging from 0.3 to 18.4 years. We performed three evaluations: evaluation 1 (M1- prenutritional intervention), M2 after 6 months, and M3 after 12 months. In these three instances we verified: the $\mathrm{z}$ score for weight/age, weight/height and height/age and the calculation of a 3-day diet record. We verified the body composition (anthropometry) in M1 and M3. The nutritional interventions were hypercaloric, hyperproteic, with adequate amount of ingested macronutrients and micronutrients.

Results: we observed an increase in the $\mathrm{z}$ score for height/age (M1=-1.07; M2=-0.69; M3=-0.50) and fat-free mass after the nutritional interventions, without improvement in the $\mathrm{z}$ score for weight/ height and fat mass. We verified an increase in the energy intake during M2 (139\%) and M3 (132\%) compared to M1 (106\%). Remarkable increase in the intake of protein, calcium, iron and vitamin $\mathrm{C}$ by the patients was found. The occurrence of anemia was found in $44 \%(8 / 18)$ of the patients.

Conclusion: the improvement of the $\mathrm{z}$ score in height/age and fat-free mass was probably due to the increase in energy consumption after the nutritional intervention. A significant improvement in the $\mathrm{z}$ score for weight/height and fat mass was not found, probably due to a gain in height and fat-free mass.

J Pediatr (Rio J) 2002; 78 (2): 161-70: cystic fibrosis, nutritional intervention, energy and nutrients, nutritional status, body composition.

1. Mestre em Nutrição pela Universidade Federal de São Paulo - Escola Paulista de Medicina

2. Médica Assistente do Setor de Pneumologia Pediátrica do Dep. de Pediatria da UNIFESP-EPM. Responsável pelo Ambulatório de Fibrose Cística do Hospital São Paulo.

3. Prof. Assistente e Chefe da Disciplina de Especialidades Pediátricas do Dep. de Pediatria da UNIFESP-EPM.

4. Profa. Titular da Faculdade de Medicina da UNISA - Faculdade de Santo Amaro.

5. Prof. Titular da Faculdade de Medicina da UNISA - Faculdade de Santo Amaro.

6. Prof. Titular da Disciplina de Nutrição e Metabolismo do Dep. de Pediatria da UNIFESP-EPM. Prof. Titular do Dep. de Pediatria da UNISA.

Artigo submetido em 12.09.01, aceito em 09.01.02. 


\section{Introdução}

A fibrose cística é caracterizada pela disfunção generalizada das glândulas exócrinas, com a produção de muco espesso e anormal, que pode obstruir os ductos dos vários órgãos afetados, causando doença pulmonar crônica, insuficiência pancreática, infertilidade masculina e concentração elevada de sódio e cloro no suor ${ }^{1}$.

Apesar do avanço no tratamento clínico e nutricional dos últimos anos, os pacientes com fibrose cística continuam a apresentar uma incidência significante de desnutrição e déficit de crescimento. A Fundação de Fibrose Cística dos Estados Unidos apontou que, em 1997, 24\% dos pacientes encontravam-se abaixo do percentil 5 para peso e $19 \%$ para estatura $^{2}$. A função pulmonar e o estado nutricional possuem íntima correlação ${ }^{3}$, sendo que a acentuada perda de peso pode levar à diminuição de massa magra, com conseqüências sobre os músculos respiratórios e elasticidade pulmonar ${ }^{4}$. Assim, a desnutrição energético-protéica é reconhecida como o pior fator prognóstico da doença ${ }^{5-8}$.

A desnutrição e o déficit de crescimento ocorrem devido à dificuldade desses pacientes em alcançar as necessidades energéticas e às perdas de energia causadas pela doença, que se devem principalmente às inflamações pulmonares crônicas e infecções ${ }^{3}$, má absorção causada pela insuficiência pancreática ${ }^{9}$, alteração na circulação enteroepática de sais biliares e antibioticoterapia ${ }^{10}$. A desnutrição pode ser agravada pela presença de anorexia, decorrente de refluxo gastroesofagiano e/ou tosse, infecções respiratórias crônicas e estresse psicossocial ${ }^{11}$. O tratamento dietético tem o papel de melhorar ou manter adequado o estado nutricional do paciente e quebrar o ciclo vicioso desnutrição-infecção.

O principal objetivo do trabalho foi avaliar, por estudo antropométrico e de composição corporal, o impacto da intervenção nutricional em pacientes com fibrose cística, com e sem insuficiência pancreática associada, em estudo longitudinal.

\section{Métodos}

Foram estudados os pacientes do Ambulatório de Fibrose Cística do Setor de Pneumologia Pediátrica da Escola Paulista de Medicina/Universidade Federal de São Paulo. A casuística compreendeu 18 pacientes, que tiveram pelo menos quatro consultas em 12 meses, com idade inicial ao acompanhamento entre 0,3 e 18,4 anos, sendo 13 do sexo masculino e 5 do feminino. Os pacientes foram acompanhados por equipe multiprofissional, composta por nutricionista, pneumopediatras, gastropediatra e fisioterapeutas.

O diagnóstico de fibrose cística foi realizado na presença de pelo menos uma das manifestações clínicas características da doença ${ }^{12}$, confirmado pela análise iônica de suor, com, no mínimo, duas amostras de sódio e cloropositivas $^{13}$, e pela detecção do gene da doença por teste genético.
O estudo incluiu coleta de dados no período de janeiro de 1998 a novembro de 2000. As avaliações foram realizadas em 3 momentos. O momento inicial (M1) ocorreu em fase pré-intervenção nutricional, o momento 2 (M2) após o período médio de 6 meses, e o momento 3 (M3) após o período médio de 12 meses (avaliação final). $\mathrm{O}$ protocolo de pesquisa foi aprovado pela Comissão de Ética Médica da instituição. Anteriormente ao estudo, os responsáveis assinaram um termo de consentimento autorizando a participação do paciente na pesquisa.

\section{Dados coletados retrospectivamente pelo prontuário}

Escore de Shwachman: realizado pelo pneumopediatra no período inicial (M1) e final (M3) do acompanhamento nutricional. É considerado método fidedigno para a avaliação da gravidade da fibrose cística. Avalia, em pontos com pesos iguais, os seguinte itens: atividade geral, dados do exame físico relacionados ao quadro pulmonar, achados radiológicos e condição nutricional ${ }^{14}$. Apesar da existência de outros testes elaborados para avaliação clínica desses pacientes, o escore de Shwachman é o que o serviço utiliza.

Colonização pulmonar: verificou-se a presença de colonização por Pseudomonas aeruginosa e Staphylococcus aureus. Foi considerado colonização crônica a persistência da bactéria durante um período maior que 6 meses. Indicouse, para os pacientes que apresentaram colonização crônica por $P$. aeruginosa, a profilaxia com antibioticoterapia a cada 3 meses, por um período mínimo de 14 dias, alternando tratamento com internação hospitalar e ambulatorial.

Internações: durante o período de acompanhamento, coletou-se dados referentes às causas das mesmas.

Hemoglobina e hematócrito: foram coletados no início do acompanhamento, utilizando-se as recomendações da World Health Organization ${ }^{15}$ para pontos de corte.

\section{Dados coletados prospectivamente}

\section{Antropometria}

$\mathrm{O}$ peso e a altura foram mensurados em todos os momentos segundo as técnicas mencionadas por Frisancho ${ }^{16}$.

- Peso: as crianças até 2 anos foram pesadas em balanças digitais pediátricas, e as crianças maiores de 2 anos, em balança para adulto, marca Filizola, sem sapatos e com indumentária mínima.

- Comprimento/altura: as crianças com até 2 anos foram medidas em antropômetro horizontal de madeira, e as crianças maiores de 2 anos, em estadiômetro de madeira, de $200 \mathrm{~cm}$ de extensão.

- Circunferência braquial (CB) e prega cutânea tricipi$\operatorname{tal}(P C T)$ : medidas nos momentos $1 \mathrm{e} 3$, no braço direito do paciente, segundo as técnicas mencionadas por Deurenberg et al. ${ }^{17}$ e pela $\mathrm{WHO}^{18}$. A PCT foi medida com o compasso de dobras cutâneas, o Lange skinfold caliper (Cambridge Scientific Industries, Cambridge, $M d$ ) e a CB, com fita métrica inextensível. 


\section{Composição corporal}

Por meio dos valores das medidas da CB e da PCT foi possível avaliar a massa adiposa e muscular, calculando-se a área muscular do braço (AMB), a área de gordura do braço (AGB) e o percentual de gordura corpora ${ }^{16}$. Para os pacientes menores de 1 ano, acompanhou-se os valores, sem classificá-los, devido a não haver dados referentes na tabela consultada.

\section{Classificação do estado nutricional}

Os dados obtidos foram avaliados em escore $\mathrm{Z}$ pelos indicadores peso/idade, peso/estatura e estatura/idade. $\mathrm{O}$ indicador peso/estatura também foi expresso como porcentagem de adequação, segundo a mediana da população de referência, como recomendado pelo Consenso de Fibrose Cística (ANTHRO, Software for Calculating Pediatric Anthropometry, v.1,02, 1999). O padrão antropométrico de referência foi o do National Center for Health Statistics ${ }^{19}$.

\section{Intervenção nutricional}

O trabalho de educação nutricional foi realizado mensalmente e individualmente e, para os pacientes estáveis do ponto de vista clínico e nutricional, bimestralmente.

\section{Monitorização nutricional}

Foi preconizado aos pacientes, conforme especificação do Consenso de Fibrose Cística, o valor energético de 120 a $150 \%$ das RDAs (Recommended Dietary Allowances, 1989 $)^{20}$ para idade e sexo e, se necessário, $45 \%$ do valor energético total em lipídios ${ }^{21}$. Orientou-se a adequação mínima de $80 \%$ das RDAs, para idade, para ferro, cálcio, zinco, vitamina $\mathrm{C}^{22}$ e $100 \%$ para proteína ${ }^{23-25}$. Nos 3 momentos do acompanhamento, calculou-se o inquérito do registro de 3 dias ou na falta deste, o do dia alimentar habitual pelo programa de informática CIS/EPM ${ }^{26}$, que analisou a proporção de macronutrientes, micronutrientes e o valor energético da alimentação.

Suplementação energética via oral: indicada aos pacientes com a relação peso/estatura $<85 \%$ ou na diminuição da velocidade do ganho de peso.

a) Suplementação lipídica: com óleo vegetal cru ou triglicerídios de cadeia média, fracionado nas principais refeições, com a dose inicial média de 16g/dia, sendo aumentada, se necessário, para $24 \mathrm{~g} /$ dia.

b) Suplementação dietética: via oral com o uso de suplemento industrializado, misturado à água ou leite.

Adesão ao suplemento nutricional via oral: foi considerada adesão o consumo diário na quantidade orientada e por um período mínimo de 6 meses.

Adesão às enzimas pancreáticas: nas consultas nutricionais, foi reforçado o uso correto das enzimas pancreáticas e sua importância para a melhora nutricional. Conside- rou-se como não-adesão aqueles pacientes que seguiram de maneira incorreta e insuficiente a ingestão das enzimas em algum momento do acompanhamento.

\section{Estudo estatístico}

Com o objetivo de comparar, para cada paciente, os resultados dos momentos 1, 2 e 3, para as variáveis consideradas, utilizou-se: a análise de variância por postos de Friedman ${ }^{27}$, e quando houve diferença significante, esta análise foi complementada pelo teste de comparações múltiplas $^{28}$. O Teste de Wilcoxon ${ }^{27}$ foi utilizado com o propósito de comparar, para cada paciente, os resultados observados nos períodos inicial (M1) e final (M3) em relação à composição corporal. O Teste $\mathrm{G}$ de $\mathrm{Cochran}^{27}$ estudou a adequação (+) nos 3 períodos do estudo. Em todos os testes fixou-se em 0,05 ou $5 \%$ o nível para a rejeição da hipótese de nulidade, assinalando-se com um asterisco os valores significantes.

\section{Resultados}

Durante o período de acompanhamento, onze pacientes $(61,1 \%)$ sofreram episódios de colonização por $S$. aureus, cinco $(27,8 \%)$ por $P$. aeruginosa e outros cinco $(27,8 \%)$ por $P$. aeruginosa crônica (Figura 1).

Entre os períodos M1 e M2, quatro pacientes foram internados, sendo destes, três lactentes. Três realizaram descontaminação para $P$. aeruginosa, e um lactente teve duas internações devido suboclusão intestinal e uma cirurgia em razão de bridas intestinais. Entre os períodos M2 e M3, sete pacientes foram internados, sendo seis para descontaminação da $P$. aeruginosa e, dentre estes pacientes, dois sofreram mais uma internação no período, uma por BCP e uma para descontaminação por S. aureus. Houve a internação de um paciente pela piora do quadro pulmonar (Figura 1).

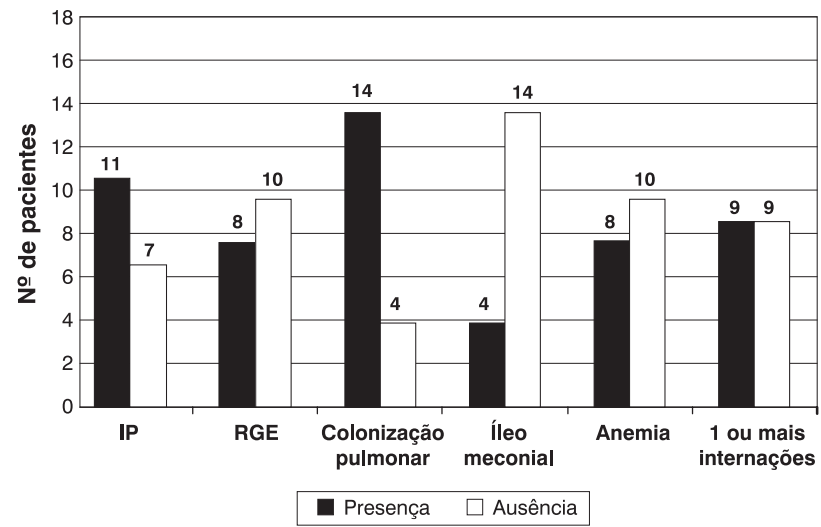

Figura 1 - Pacientes com fibrose cística e quadro clínico presente durante o período de acompanhamento (M1M2-M3) 
Houve aumento significante nos valores do escore de Schwachman ao final do acompanhamento em relação ao período pré-intervenção, não sendo verificado alteração na classificação do escore (Tabela1).

Foi observado melhora na relação do escore $\mathrm{Z}$ de E/I após a intervenção nutricional nos períodos M2 e M3 em relação ao M1. Contudo, praticamente não houve modificação no escore $Z$ de $P / E$ e ficou sem melhora significante do escore $\mathrm{Z}$ de $\mathrm{P} / \mathrm{I}$ após a intervenção (Tabela 2).

Tabela 1 - Pacientes com fibrose cística, segundo a média e mediana do escore de Shwachman nos períodos M1 (inicial) e M3 (final) do acompanhamento. Resultado do teste de Wilcoxon

\begin{tabular}{lccc}
\hline Período & M1 & M3 & M1 $\mathbf{x}$ M3 \\
\hline Média & 76,8 & 82,8 & T calc: $13,5^{*}$ \\
Mediana & 77,5 & 85,0 & $\mathrm{p}<0,02$ \\
\hline
\end{tabular}

Verificou-se aumento significante na massa magra dos pacientes, representada pela AMB, no M3 em relação ao M1, porém sem melhora na AGB e no percentual de gordura corporal (Tabela 3).

Houve melhora significante no consumo de energia no M2 em relação ao M1. Analisando-se os micronutrientes, verificamos que houve melhora significante no consumo de cálcio do M2 em relação ao M1. No consumo de ferro, houve melhora significante, porém essa melhora não foi observada quando analisamos o número de pacientes adequados em ferro. Não se verificou melhora significante no consumo de zinco e vitamina $\mathrm{C}$, no entanto, para a vitamina $\mathrm{C}$, houve considerável aumento na média da vitamina consumida do M2 e M3 em relação ao M1 (Tabelas 4 e 5).
Tabela 3 - Média e mediana da composição corporal dos pacientes com fibrose cística analisada pela área muscular do braço (AMB), área de gordura do braço (AGB) e percentual de gordura corporal nos momentos M1 (inicial) e M3 (final). Resultado da análise do teste de Wilcoxon

\begin{tabular}{lcccc}
\hline & M1 & M3 & Z calculado & Significância \\
\hline AMB & 16,58 & 20,83 & $3,55^{*}$ & M1<M3 \\
AGB & 6,48 & 7,47 & 1,81 & NS \\
\% Gordura & 27,57 & 26,07 & 1,54 & NS \\
\hline
\end{tabular}

${ }^{*} p<0,001$

\section{Discussão}

Centenas de mutações relacionadas à fibrose cística já foram descritas e se acompanha de grande variabilidade no quadro clínico. A mutação DF508 é a mais freqüente entre a população caucasiana, e, quando homozigoto, em geral têm sido fortemente associada à insuficiência pancreáti$\mathrm{ca}^{29}$. Neste estudo, dentre os onze pacientes com insuficiência pancreática, nove apresentaram a mutação DF508 e, dentre os suficientes pancreáticos ${ }^{7}$, apenas dois tiveram esta mutação num dos cromossomos. Em relação à manifestação da doença pulmonar, estudos não têm comprovado associação com o genótipo ${ }^{29}$.

Os dados levantados demonstraram a presença da mutação genética DF508 homozigoto em quatro $(22,2 \%)$ pacientes, e sete $(38,9 \%)$ apresentaram a mutação genética DF508 heterozigoto. Outros estudos realizados no Brasil indicam a presença desta mutação entre $27 \%$ e $53 \%$ dos pacientes ${ }^{30-34}$. A presença da mutação DF508 varia conforme a incidência da população causacóide, o que levou Raskin et al. a afirmarem que a freqüência desta mutação no Brasil é parecida com a dos países do sul da Europa, conforme a imigração européia no período de colonização do Brasil ${ }^{31}$.

Tabela 2 - Pacientes com fibrose cística, segundo os indicadores nutricionais durante o seguimento (M1, M2 e M3). Resultado da Análise de variância por postos de Friedman

\begin{tabular}{lccccc}
\hline & M1 & M2 & M3 & $\boldsymbol{\chi}^{\mathbf{2} \text { calculado }}$ & Significância \\
\hline \% P/E & 94,35 & 96,65 & 93,90 & 3,44 & NS \\
P/E escore z & $-0,83$ & $-0,58$ & $-0,80$ & 1,36 & NS \\
P/I escore z & $-1,36$ & $-0,93$ & $-0,90$ & 4,00 & NS \\
E/I escore z & $-1,07$ & $-0,69$ & $-0,50$ & $11,37^{*}$ & M1 < M2 e M3 \\
\hline
\end{tabular}

${ }^{*} p<0,01$ 
Tabela 4 - Pacientes com fibrose cística, segundo o consumo de energia e de nutrientes durante o seguimento (M1, M2 e M3). Resultado da análise de variância por postos de Friedman

\begin{tabular}{|c|c|c|c|c|c|}
\hline & M1 & M2 & M3 & $\chi^{2}$ calculado & Significância \\
\hline$\%$ Energia/RDAs & 106 & 139 & 132 & $9,00 *$ & $\mathrm{M} 1<\mathrm{M} 2$ \\
\hline$\%$ Proteína/RDAs & 224 & 290 & 303 & 5,77 & NS \\
\hline$\%$ Lipídios/VCT ${ }^{\dagger}$ & 32,8 & 36,5 & 36,4 & 3,44 & NS \\
\hline$\%$ Carboidrato/VCT ${ }^{\dagger}$ & 55,3 & 51,0 & 50,5 & 2,11 & NS \\
\hline$\%$ Proteína/VCT ${ }^{\dagger}$ & 11,9 & 12,5 & 13,1 & 2,11 & NS \\
\hline \% Cálcio/RDAs & 75,2 & 108,3 & 92,7 & $8,77 *$ & $\mathrm{M} 1<\mathrm{M} 2$ \\
\hline$\%$ Zinco/RDAs & 89,8 & 90,8 & 99,4 & 0,25 & NS \\
\hline$\%$ Ferro/RDAs & 102,5 & 133,7 & 141,2 & $8,44^{*}$ & $\mathrm{M} 1<\mathrm{M} 2$ e $\mathrm{M} 3$ \\
\hline$\%$ Vit. C /RDAs & 140,4 & 285,3 & 490,8 & 5,44 & NS \\
\hline
\end{tabular}

${ }^{*} p<0,02 \quad+$ valor calórico total

A P. aeruginosa é a principal bactéria responsável pelo progressivo dano pulmonar. A incidência de $P$. aeruginosa aumenta com a idade e atinge de 70 a $90 \%$ dos pacientes ${ }^{35}$. Estudos verificaram perda de peso quando há colonização pulmonar por P.aeruginosa, contudo o mecanismo pelo qual ocorre esta perda ainda não está esclarecido ${ }^{6,36}$. Num estudo realizado com ratos, também observou-se perda de peso quando há colonização pulmonar aguda por P. aeruginosa, sendo esta perda correlacionada à produção de citoquinas do estado inflamatório, e não com a alteração da resposta pulmonar ${ }^{37}$. Neste estudo, em todas as faixas etárias, houve a presença de episódios de colonização pulmonar por P.aeruginosa e S.aureus durante o acompanhamento, sendo que em pacientes maiores de nove anos observou-se maior ocorrência de colonização crônica por P.aeruginosa (4/5) (Figura 1). A colonização nem sempre causou diminuição do apetite e conseqüente perda de peso, principalmente para aqueles que apresentavam bom estado nutricional. De modo geral, como a desnutrição encontrada foi leve, e os pacientes mostraram melhora nutricional durante o acompanhamento, a colonização pulmonar por P.aeruginosa influenciou o peso da maior parte dos pacientes. Shale confirma estas evidências, referindo que desnutrição crônica aumenta o risco de colonizações pulmonares e afeta a resposta imune ${ }^{38}$.

A internação dos pacientes por si só não foi necessariamente correlacionada com a piora do estado nutricional,

Tabela 5 - Pacientes com fibrose cística, segundo a média do percentual adequação (+) do consumo de energia, cálcio, zinco, ferro e vitamina $\mathrm{C}$ referente às RDAs para idade nos 3 momentos do acompanhamento. Resultado da análise do teste do G de Cochran

\begin{tabular}{lccccc}
\hline & M1 & M2 & M3 & G calculado & Significância \\
\hline Adequação/Energia n (+) & 7 & 13 & 9 & $6,22^{*}$ & M1 < M2 \\
\% (+) & 38,9 & 72,2 & 50,0 & & \\
Adequação/Cálcio n (+) & 6 & 11 & 10 & $6,00^{*}$ & M1 < M2 \\
$\%(+)$ & 33,3 & 61,1 & 55,6 & & \\
Adequação/Zinco n (+) & 10 & 10 & 12 & 0,89 & NS \\
$\%(+)$ & 55,6 & 55,6 & 66,7 & & \\
Adequação/Ferro n (+) & 13 & 15 & 12 & 1,40 & NS \\
$\%(+)$ & 72,2 & 83,3 & 66,7 & & NS \\
Adequação/Vit. C n (+) & 8 & 12 & 12 & 3,56 & \\
$\%(+)$ & 44,4 & 66,7 & 66,7 & & \\
${ }^{*}$ p<0,05 & & & & &
\end{tabular}


pois neste estudo a maioria dos pacientes sofreu internação casual ou programada para descolonização da $P$. aerugino$s a$, não perdendo peso nem antes e durante o período da descolonização (Figura 1).

Em nosso estudo, demonstramos melhora significante na relação estatura/idade após a intervenção nutricional contínua via oral. Não houve alterações significantes no escore $\mathrm{Z}$ de peso/estatura, durante o acompanhamento. Houve aumento da relação peso/idade durante o seguimento, embora não significante (Tabela 2).

Recente estudo longitudinal realizado no Brasil, com amostra maior que a deste trabalho, observou aumento significante no escore $\mathrm{Z}$ de P/I, após 13 e 43 meses de intervenção nutricional contínua via oral, para pacientes de 6 meses a 18 anos. Contudo, os resultados foram parecidos com os do presente estudo para os pacientes menores de 5

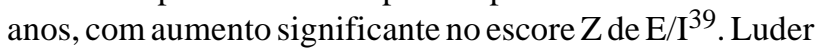
et al. não verificaram melhora significante no escore $Z$ para estatura, em pacientes com idades entre 2 e 27 anos, com aumento significante de peso, após a intervenção nutricional contínua, via oral ${ }^{40}$.

O estudo de Gaskin et al. tentou promover a melhora do estado nutricional por meio da suplementação nutricional via oral, sem resultados significativos. Todavia, no grupo (4-16 anos) em que foi introduzida a dieta noturna por gastrostomia foi verificado melhora significante nos indicadores nutricionais, após 18 meses de intervenção ${ }^{41}$. O'Loughin et al. somente observaram melhora significante no peso, massa magra e gorda após a intervenção por sonda nasoenteral por 6 meses, em pacientes (7-27 anos) que não obtiveram melhora com a suplementação via oral ${ }^{42}$.

Outros estudos que realizaram intervenção nutricional agressiva, por período mínimo de 12 meses, com dieta noturna por gastrostomia, demonstraram melhora no estado nutricional. Levy et al. observaram aumento significante na estatura e peso de pacientes entre 4,9 e 21,5 anos ${ }^{5}$. Outros trabalhos referiram aumento significante do peso para pacientes maiores de 7 anos $^{43,44}$. De maneira geral, os estudos que realizaram suplementação via oral apresentaram dificuldade em melhorar o estado nutricional dos pacientes, tendo a intervenção nutricional agressiva resultados mais positivos sob este ponto de vista.

Neste estudo, os lactentes apresentaram melhora mais acentuada no indicadores antropométricos e na composição corporal após a intervenção nutricional, em relação às demais faixas etárias. Este grupo tinha, ao início do acompanhamento (M1), o maior déficit de estatura e de peso. De modo parecido, outros estudos comprovaram a recuperação estatural parcial ou total nos primeiros anos de vida, que pode ocorrer com maior velocidade quando a intervenção nutricional é precoce. Este fato realça a importância do diagnóstico precoce, por meio do screening neonatal, ao nascimento ${ }^{45-49}$, com a dosagem da tripsina imuno-reativa no sangue ${ }^{50}$.
Demonstramos melhora significante na área muscular do braço (AMB) após a intervenção nutricional, porém nem todos os pacientes alcançaram o percentil 5 da tabela de Frisancho, que é considerado o mínimo adequado ${ }^{16}$. A massa gorda e o percentual de gordura corporal apresentaram-se sem alterações significantes durante o seguimento (Tabela 3). Estudos que compararam os pacientes a um grupo controle observaram que crianças ${ }^{51-53}$, adolescentes $^{52}$ e adultos ${ }^{54-56}$ possuem valores abaixo do recomendado para massa magra e gorda, reforçando a idéia de que, mesmo com a melhora da composição corporal, não são todos os pacientes que conseguem atingir a normalidade, como o observado.

De modo similar a este estudo, Greer et al. verificaram melhora na composição corporal de lactentes diagnosticados ao screening neonatal, após intervenção nutricional contínua até os 12 meses de idade, com tendência à menor massa gorda ${ }^{45}$. Estudos que realizaram dieta noturna por gastrostomia, pelo período mínimo de 12 meses, observaram não só melhora significante na massa magra, mas, também, aumento significante na massa gorda ${ }^{5,43,44}$.

Verifica-se, portanto, que a recuperação nutricional é diferenciada, isto é, enquanto estudos comprovam melhora estatural ou da massa magra, outros demonstram aumento de peso e da massa gorda ou de ambos. Neste estudo, houve desvio da energia consumida para a promoção do ganho estatural, com aumento da velocidade de crescimento e da massa magra, também porque os pacientes já apresentavam, de início, boa relação peso/estatura. Imaginamos que deva haver, além dos fatores quantitativos, outros fatores, qualitativos, na alimentação, que promovam o ganho de massa magra e da estatura. Para Abrams, os nutrientes antioxidantes podem interferir no quadro pulmonar e no estado nutricional ${ }^{57}$.

Neste estudo, verificamos que em média os pacientes atingiram o percentual recomendado em energia após a intervenção nutricional (Tabela 4). Todavia, em relação ao percentual de pacientes adequados, assim como em outros trabalhos, relata-se a dificuldade em alcançar a recomendação de $120 \%$ de energia e $40 \%$ em lipídios para todos os pacientes ${ }^{40,58-61}$ (Tabela 5). Alguns autores afirmam que não é necessário atingir 120\% das RDAs (Recommended Dietary Allowances) em energia em todas as fases da doença ${ }^{46,62,63}$. Wooton et al. assinalam que as RDAs são calculadas referentes à idade e não ao peso, sugerindo que as necessidades energéticas são superestimadas ${ }^{64}$. É possível que em nosso estudo alguns dos pacientes não necessitassem atingir $120 \%$ de energia, devido a menor existência de indivíduos insuficientes pancreáticos $(61 \%)$ (Figura 1) do que a referida na literatura ( $85 \%$ ), e ao predomínio de pacientes com quadro clínico leve. Segundo os resultados do escore de Shwachman, os pacientes mantiveram-se estáveis durante o período de acompanhamento, evidenciando-se aumento estatisticamente significante da média e mediana entre M1 e M3, mesmo não havendo mudança na 
classificação do escore. Esses resultados mostram que o tratamento clínico e nutricional foram eficazes durante o período de acompanhamento, sendo esse escore um dos mais utilizados para a avaliação do quadro clínico nos principais centros de tratamento para a fibrose cística (Tabela 1). Numa comparação descritiva, verificou-se que dentre os cinco lactentes estudados, quatro, ao final do acompanhamento, apresentaram a classificação excelente do escore de Shwachman, com alteração positiva do escore. As demais faixas etárias não mostraram esta mesma intensidade de mudança.

Nos retornos entre os momentos 1, 2 e 3 do acompanhamento, foi reforçada a orientação nutricional ao paciente, conforme os erros alimentares ou deficiências nutricionais encontradas. Prioritariamente, para os pacientes com deficiente estado nutricional, foi indicada a adequação do esquema alimentar (número de refeições, intervalo entre as refeições, distribuição dos macronutrientes nas refeições, preparações hipercalóricas) e suplementação lipídica com óleo de soja cru.

Trabalhos têm utilizado óleos vegetais como forma de aumentar o valor energético, e o percentual de gordura e ácidos graxos essenciais na alimentação ${ }^{46,65}$. Assim, optamos, prioritariamente, pelo aumento na densidade energética com óleo de soja, já que não altera o volume ingerido, é de baixo custo e, dentre os óleos, o que possui melhor proporção dos ácidos graxos ômega 3 e $6^{66}$. Houve boa adesão quanto ao uso das enzimas pancreáticas e da suplementação com óleo de soja, em função do trabalho multidisciplinar ter proporcionado um bom vínculo entre a equipe e o paciente (Figura 2). O controle referente à adesão ao uso do suplemento nutricional e às enzimas foi realizado em todos os retornos nutricionais, na anamnese feita junto ao paciente e acompanhante.
Demonstrou-se aumento na média de proteína consumida nos momentos 2 e 3 que, contudo, já no M1, ultrapassava as RDAs, com percentual proporcionalmente adequado (10-15\%) em relação ao valor energético total (Tabela 4). Outros estudos indicam, do mesmo modo, que o consumo protéico costuma ultrapassar facilmente as RDAs para idade ${ }^{40,41,46,58}$.

O número de pacientes com ingestão adequada de cálcio praticamente dobrou no M2 e M3 em relação ao M1. Não foram observadas alterações significantes no consumo médio de zinco e no número de pacientes adequados em zinco durante o seguimento. Houve aumento significante no consumo de ferro, no M2 e M3, em relação ao M1. Contudo, o número de pacientes adequados praticamente manteve-se $\mathrm{o}$ mesmo durante o seguimento. A dificuldade para que os pacientes atingissem o recomendado para zinco e ferro pode ter ocorrido devido aos momentos de anorexia, em que as refeições mais comprometidas eram o almoço e o jantar, justamente onde há maior consumo de ferro e zinco. Anemia foi encontrada em $8(44,4 \%)$ pacientes durante o período de acompanhamento, 3 em lactentes e 5 em préescolares e escolares, sendo que nestes dois últimos a anemia foi leve (Figura 1). Houve tendência para a melhora no consumo de vitamina $C$, nos momentos $2 \mathrm{e} 3$ em relação ao M1, com considerável aumento no número de pacientes adequados após a intervenção nutricional (Tabelas 4 e 5).

Kawchak et al. mencionam que, de modo geral, atingese o recomendado para os micronutrientes de acordo com as RDAs para pacientes com fibrose cística ${ }^{58}$. Todavia, os estudos são escassos e dificilmente referem o percentual de pacientes adequados em micronutrientes e energia consumidos, e costumam apenas apresentar a média consumida, que pode estar adequada, porém não refletindo a realidade de casos individuais.

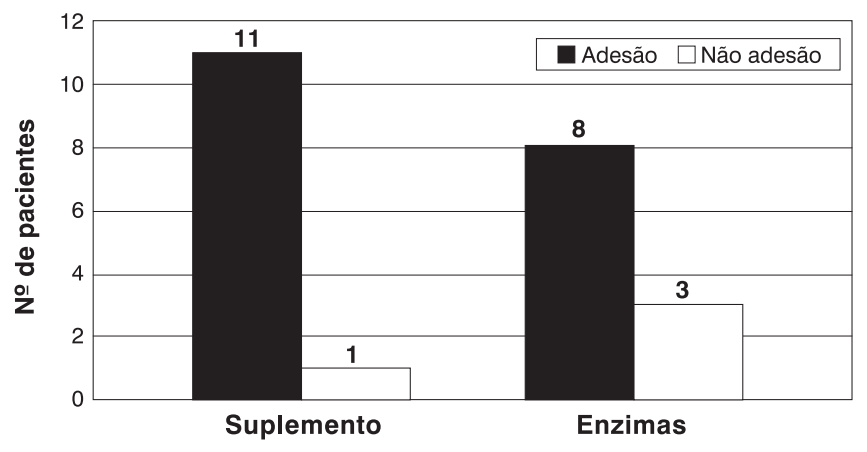

Figura 2 - Pacientes com fibrose cística e adesão ao uso de suplemento nutricional e de suplementação enzimática 
As limitações do estudo, como o tamanho amostral, variabilidade do quadro clínico e da faixa etária, não possibilitaram análises estatísticas mais detalhadas para comparações entre subgrupos. Pelo pequeno número de estudos longitudinais sobre o estado nutricional, composição corporal e consumo alimentar nesta patologia, acreditamos justificar-se sua divulgação, mesmo com as limitações, que são inerentes ao nosso meio.

Resumindo, a intervenção nutricional oferecida aos pacientes com fibrose cística mostrou que a energia fornecida pela alimentação foi utilizada prioritariamente para aumento da velocidade de crescimento, demonstrada pela melhora da relação estatura/idade e da massa magra no período pós-intervenção, sendo que os lactentes apresentaram melhora mais acentuada nos indicadores nutricionais em relação às demais faixas etárias. Concluiu-se que os lactentes possuíram maior capacidade de melhora nutricional dentre as demais faixas etárias, que pode ter sido em razão do menor comprometimento pulmonar, apetite voraz, favorecendo a eficácia do tratamento clínico e nutricional. Não houve alteração em análise total dos pacientes da relação peso/estatura e da massa gorda, indicando que o ganho estatural foi prioritário em relação ao ganho ponderal. A adesão à suplementação nutricional e ao uso correto de enzimas pancreáticas e a melhora no consumo de micronutrientes também auxiliou a melhora nutricional.

O trabalho de educação nutricional é necessário para um melhor prognóstico do paciente, sendo mais eficaz quando realizado seqüencialmente e com bom vínculo entre equipe e paciente. A monitorização do estado nutricional, aliada aos avanços no tratamento clínico e fisioterápico, o diagnóstico precoce e a maior eficácia dos medicamentos, induzem à melhora na sobrevida atual dos pacientes.

\section{Referências bibliográficas}

1. MacLusky I. Cystic fibrosis for the primary care pediatrician. Pediatric Annals 1993;22:9.

2. Zemel BS, Jawad AF, Fitzsimmons S, Stallings VA. Longitudinal relationship among growth, nutritional status, and pulmonary function in children with cystic fibrosis: analysis of the Cystic Fibrosis Foundation National CF Patient Registry. J Pediatr 2000;137:374-80.

3. Elborn JS, Bell SC. Nutrition and survival in cystic fibrosis. Thorax 1996;51:971-2.

4. Bentur L, Kalnins D, Levison H, Corey M, Durie PR. Dietary intakes of young children with cystic fibrosis: is there a difference? J Pediatr Gastroenterol Nutr 1996;22:254-8.
5. Levy LD, Durie PR, Pencharz PB, Corey ML. Effects of longterm nutritional rehabilitation on body composition and clinical status in malnourished children and adolescents with cystic fibrosis. J Pediatr 1985;107:225-30.

6. Shepherd RW, Vasques-Velasquez L, Prentice A, Holt TL, Coward WA, Lucas A. Increased energy expenditure in children with cystic fibrosis. Lancet 1988;1:1300-3.

7. Kerem E, Reisman J, Corey M, Canny G, Levinson H. Prediction of mortality in patients with cystic fibrosis. N Engl J Med 1992;32:1187-91.

8. Roulet M. Protein-energy malnutrition in cystic fibrosis patients. Acta Paediatr 1994;395 Suppl :42-48.

9. Levine JJ. Nutritional supplementation in cystic fibrosis: are all patients candidates for aggressive therapy? J Pediatr Gastroenterol Nutr 1998;21:120-1.

10. Reilly JJ, Edwards CA, Weaver LT. Malnutrition in children with cystic fibrosis: the energy-balance equation. J Pediatr Gastroenterol Nutr 1997;25:127-36.

11. Adams E. Cuidado nutricional na doença pulmonar. In: Mahan LK, Arlin MT. Alimentos, Nutrição e Dietoterapia. $8^{a}$ ed. São Paulo: Rocca; 1995. p.620-3.

12. Rosenstein BJ, Cutting GR. The diagnosis of cystic fibrosis: a consensus statement. J Pediatr 1998;132:589-95.

13. Gibson LE, Cooke R. A test for concentration of electrolytes in sweat in cystic fibrosis of the pancreas utilizing pilocarpine by iontophoresis. Pediatrics 1959; 23:545-9.

14. Shwachman H, Kulczycki LL. Long term study of one hundred five patients with cystic fibrosis. Am J Dis Child 1958;96:6-15.

15. World Health Organization. Nutritional Anemia. Geneva, WHO Expert Committee; 1972.

16. Frisancho AR. Anthropometric standards for the assessment of growth and nutritional status. Michigan: The University of Michigan Press; 1990

17. Deurenberg P, Pieters JJL, Hautvast JGAJ. The assessment of the body fat percentage by skinfold thickness measurements in childhood and young adolescence. Br J Nutr 1990;63:293-303.

18. World Health Organization. Physical status: the use and interpretation of anthropometry. Geneva, WHO Expert Committee; 1995.

19. World Health Organization. Measuring change in nutritional status: guidelines for assessing the nutritional impact of supplementary feeding programmes. Geneva; 1983.

20. Food And Nutrition Board. Recommended Dietary Allowances. 10th ed. Washington, DC: National Academy of Sciences; 1989.

21. Ramsey BW, Farrell PM, Pencharz P. Consensus Committee. Nutritional Assessment and Management in Cystic Fibrosis: a Consensus Report. Am J Clin Nutr 1992;55:108-16.

22. Krebs-Smith SM, Clarck LD. Validation of a nutrient adequacy score for use with women and children. J Am Diet Assoc 1989;89:775-80.

23. Durie PR, Pencharz PB. Nutritional deficiencies in cystic fibrosis. Br Med Bull 1992;48:823-47.

24. Strandvik B. Tratamento nutricional na mucoviscidose. Anais Nestlé; 1993. p.37-46.

25. Green MR, Buchanan E, Weaver LT. Nutritional management of the infant with cystic fibrosis. Arch Dis Child 1995;72:452-6.

26. Anção MS, Cuppari L, Tudisco ES, Draibe AS, Sigulem DM. Sistema de Apoio à Nutrição [programa de computador]. Versão 2.5a. São Paulo: Centro de Informática em Saúde, Universidade Federal de São Paulo; 1993. 
27. Siegel S, Castellan Jr NJ. Nonparametric Statistics. 2nd ed. New York: McGraw-Hill; 1988.

28. Hollander M, Wolfe DA. Nonparametric Statistical Methods. New York: John Wiley \& Sons; 1973. p.503.

29. Kerem E, Kerem B. Genotype - Phenotype correlations in cystic fibrosis. Pediatr Pulmonol 1996;22:387-95.

30. Martins CSB, Ribeiro F, Costa FF. Frequency of the cystic fibrosis DF508 mutation in a population from São Paulo State, Brazil. Braz. J Med Biol Res 1993;26:1037-40.

31. Raskin S, Phillips JA, Krishnamani MRS, Vnencak-Jones C, Parker RA, Rozov T, et al. DNA analysis of cystic fibrosis in Brazil by direct PCR amplification from Guthrie cards. Am J Med Gen 1993;46:665-9.

32. Damasceno N. Correlação do genótipo DF508 e fenótipo pulmonar em 40 pacientes com fibrose cística [dissertação]. São Paulo: Faculdade de Ciências Médicas da Santa Casa de São Paulo; 1996.

33. Maróstica PJC, Raskin S, Abreu-Silva FA. Analysis of the DF508 mutation in a Brazilian cystic fibrosis population: comparison of pulmonary status of homozygotes with other patients. Braz J Med Biol Res 1998;31:529-32.

34. Reis FJC, Oliveira MCL, Penna FJ, Oliveira GR, Oliveira EA, Monteiro AF. Quadro clínico e nutricional de pacientes com fibrose cística: 20 anos de seguimento no HC-UFMG. Rev Ass Med Brasil 2000;46(4):325-30.

35. Ruzal-Shapiro C. Cystic Fibrosis. Radiol Clin North Am 1998;36:143-61.

36. Naon H, Hack S, Shelton MT, Gotthoffer RC, Gozal D. Resting energy expenditure: evolution during antibiotic treatment for pulmonary exacerbation in cystic fibrosis. Chest 1993;103: 1819-25.

37. Heeckeren AM, Tscheikuma J, Walenga RW, Konstan MW, Davis PB, Erokwu B, et al. Effect of Pseudomonas infection on weight loss, lung mechanics, and cytokines in mice. Am J Respir Crit Care Med 2000;161:271-9.

38. Shale DJ. Nutrition and Immunity. Respir Med 1997;91(10):A 69-71.

39. Adde FV. Perfil nutricional de pacientes com fibrose cística: papel do aconselhamento nutricional em ambulatório [dissertação]. São Paulo: Faculdade de Medicina da USP; 2000.

40. Luder E, Kattan M, Thornton JC, Koehler KM, Bonforte RJ. Efficacy of a nonrestricted fat diet in patients with cystic fibrosis. AJDC 1989;143:458-64.

41. Gaskin KJ, Waters DL, Baur LA, Soutter VL, Gruca MA. Nutritional status, growth and development in children undergoing intensive treatment for cystic fibrosis. Acta Paeditr Scand 1990; 366 Suppl :106-10.

42. O`Loughlin E, Forbes D, Parsons H, Scott B, Cooper D, Gall G. Nutritional rehabilitation of malnourished patients with cystic fibrosis. Am J Clin Nutr 1986;43:732-7.

43. Vaisman N, Clarke R, Pencharz PB. Nutritional rehabilitation increases resting energy expenditure without affecting protein turnover in patients with cystic fibrosis. J Pediatr Gastroenterol Nutr 1991;13:383-90.

44. Steinkamp G, Hardt H. Improvement of nutritional status and lung after long-term nocturnal gastrostomy feedings in cystic fibrosis. J Pediatr 1994;124:244-9.

45. Greer R, Sherpherd R, Cleghorn G, Bowling FG, Holt T. Evaluation of growth and changes in body composition following neonatal diagnosis of cystic fibrosis. J Pediatr Gastroenterol Nutr 1991;13:52-8.

46. Marcus MS, Sondel SA, Farrell PM, Laxova A, Carey PM, Langhough R, et al. Nutritional status of infants with cystic fibrosis associated with early diagnosis and intervention. Am J Clin Nutr 1991;54:578-85.
47. Dankert-Roelse JE, Te Meerman GJ. Long term prognosis of patients with cystic fibrosis in relation to early detection by neonatal screening and treatment in a cystic fibrosis centre. Torax 1995;50:712-8.

48. Farrell PM, Kosorok MR, Laxova A, Shen G, Koscik RE, Bruns WT, et al. Nutritional benefits of neonatal screening for cystic fibrosis. N Engl L Med 1997; 337:963-9.

49. Farrell PM, Kosorok MR, Rock MJ, Laxova A, Zeng LL, et al. Early diagnosis of cystic fibrosis through neonatal screening prevents severe malnutrition and improves long-term growth. Wisconsin cystic fibrosis neonatal screening study group. Pediatrics 2001;107:1-13.

50. Hammond KB, Abman SH, Sokol RI, Accurso FJ. Efficacy of statewide neonatal screening for cystic fibrosis by assay of trypsinogen concentrations. N Engl J Med 1991;325:769-74.

51. Tomezsko JL, Scanlin TF, Stallings VA. Body composition of children with cystic fibrosis with mild clinical manifestations compared with normal children. Am J Clin Nutr 1994;59:123-8.

52. Henderson RC, Madsen CD. Bone mineral content and body composition in children and young adults with cystic fibrosis. Pediatr Pulmonol 1999;27:80-4.

53. Stettler N, Kawchak DA, Boyle LL, Propert KI, Scanlin TF, Stallings VA, et al. A prospective study of body composition changes in children with cystic fibrosis. Ann NY Acad Sci 2000;904:406-9.

54. Grey AB, Ames RW, Matthews RD, Reid IR. Bone mineral density and body composition in adult patients with cystic fibrosis. Thorax 1993;48:589-93.

55. Rochat T, Slosman DO, Pichard C, Belli DC. Body composition analysis by dual-energy $\mathrm{x}$-ray absorptiometry in adults with cystic fibrosis. Chest 1994;106:800-5.

56. Ionescu AA, Nixon LS, Evans WD, Stone MD, Lewis-Jenkins V, Chatham K, et al. Bone density, body composition, and inflammatory status in cystic fibrosis. Am J Respir Crit Care Med 2000;162(3 Pt 1):789-94.

57. Abrams SA. Chronic pulmonary insufficiency in children and its effects on growth and development. J Nutr 2001;131:938S-41S.

58. Kawchak DA, Zhao H, Scanlin TF, Tomezsko JL, Cnaan A, Stallings VA. Longitudinal, prospective analysis of dietary intake in children with cystic fibrosis. J Pediatr 1996;129:119-29.

59. Stark LJ, Mulvihill MM, Jelalian E, Bowen AM, Powers SW, Tao S, et al. Descriptive analysis of eating behavior in school-age children with cystic fibrosis and healthy control children. Pediatrics 1997;99:665-71.

60. Anthony H, Bines J, Phelan P, Paxton S. Relation between dietary intake and nutritional status in cystic fibrosis. Arch Dis Child 1998;78:443-7.

61. Westwood ATR, Saitowitz R. Growth and nutrition in South African children with cystic fibrosis. S Afr Med 1999;89:1276-8.

62. Gandarillas IM, Orejas RAG, Bousono CG, Cue RG, Ramos EP, Crespo MH. Valoración del estado nutricional en un grupo de pacientes con fibrosis quística. An Esp Pediatr 1996;44:40-4.

63. Stettler N, Kawchak DA, Boyle LL, Propert KI, Scanlin TF, Stallings VA, et al. Prospective evaluation of growth, nutritional status, and body composition in children with cystic fibrosis. Am J Clin Nutr 2000;72:407-13.

64. Wooton SA, Murphy JL, Bond SA, Ellis JE, Jackson AA. Energy balance and growth in cystic fibrosis. J R Soc Med 1991;84 Suppl 18:22-7. 
65. Rettammel AL, Marcus MS, Farrell P, Sondel SA, Koscik RE, Mischler EH. Oral supplementation with a high-fat, high-energy product improves nutritional status and alters serum lipids in patients with cystic fibrosis. J Am Diet Assoc 1995;95:454-9.

66. Shils M, Olson S, Shike M. Modern Nutrition in Health and Diseases. Philadelphia: Lea \& Febiger; 1994, p.214-30, Annex: A-100-4.
Endereço para correspondência:

Dra. Maria Cristina de Almeida Gaspar

Rua Piraçununga, 343 - Alto da Mooca

CEP 03187-010 - São Paulo, SP

Fone: 6601.3824 - Fax: 6606.4613 